Supporting Information

\title{
Spin Coupling in the Supramolecular Structure of a New Tetra(Quinoline-TEMPO) Yttrium(III) Complex
}

Luca Maretti, Marilena Ferbinteanu, Fanica Cimpoesu, Saiful S.M. Islam, Yasunori Ohba, Takashi Kajiwara, Masahiro Yamashita and Seigo Yamauchi*

yamauchi@tagen.tohoku.ac.jp, marilena@agnus.chem.tohoku.ac.jp

I. Magnetic Susceptibility Fit Details.

II. Ab Initio Calculation Details.

III. The Dipolar Spin Coupling Hamiltonian.

IV. Crystallographic Data. 


\section{Magnetic Susceptibility Fit Details.}

This section is devoted to the details illustrating the aspects of fitting of the exchange parameters with respect to experimental magnetic susceptibility. In a necessary quick summarizing we recall that the system at hand is a rectangle of spins, with $J_{a}$ parameter along the shorter edge, $J_{b}$ for the longer one and $J_{d}$ on diagonals. The simple intuition as well the reliable ab initio calculations are pointing toward a $\left|J_{a}\right|>\mid J_{b}$ $\mid$ and $J_{d} \sim 0$ expectation. However the automatic fit leads to other tendencies, as described in the text and illustrated in the figures below.

Figure I.1.a deals with the fitting of $J_{d}$ when the edges are assumed parametrically equal, $J_{a}=J_{b}=J_{e}$. The error is represented on the secondary axis. Its minimum occurs in the point of $J_{d}=J_{e}$, namely the artifactual quasi-tetrahedral parametric topology. In Figure I.1.b the constrained results of $J_{d}=0$ and optimal $J_{b}$. vs. $J_{a}$ are shown, illustrating the artifactual tendency toward a perfect square instead of the actual rectangle.

The methodological emphasis is a warning for the need to critically evaluate the primary results of the automatically fit with supplementary criteria, whenever is possible.

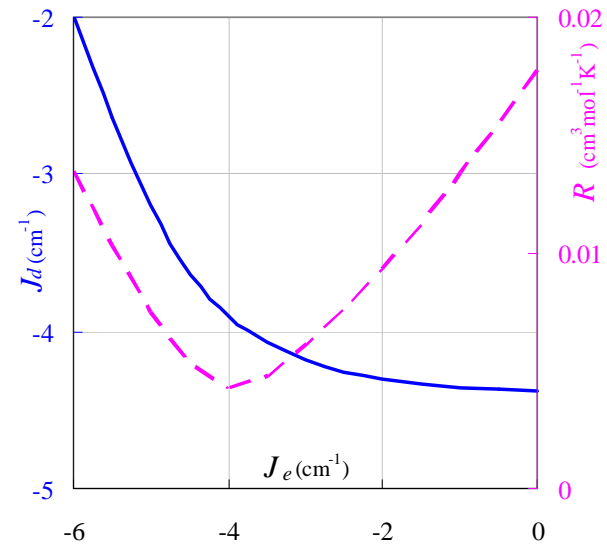

a)

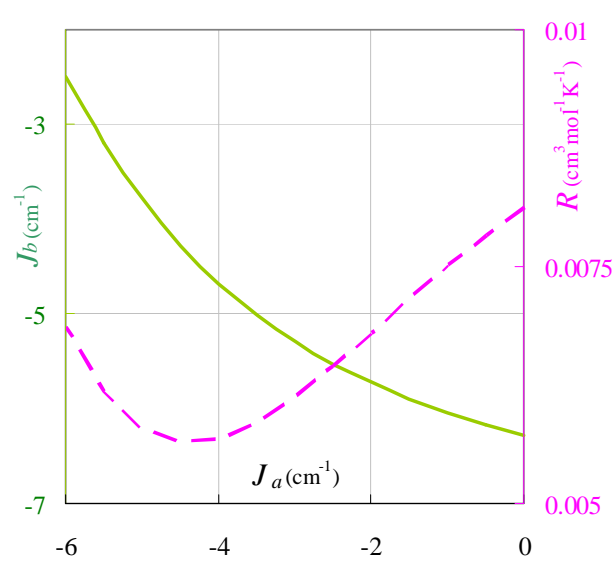

b)

Figure I.1. a) The fitting of the diagonal parameter $J_{d}$ under the assumption of square topology, when the edge parameter $J_{e}$, is varied. The resulted $J_{d}$ is shown in continuous line; the error curve $R$, relied on the secondary axis, with dashed line. b) The fitting in rectangle with imposed $J_{d}=0$; the resulted $J_{b}$ against the varied $J_{a}$ and the corresponding error $R . \quad J$ variables are in $\mathrm{cm}^{-1}$ and the absolute error $R$ is in $\mathrm{cm}^{3} \cdot \mathrm{mol}^{-1} \cdot \mathrm{K}^{-1}$. 


\section{Ab Initio Calculation Details.}

The text described the CASSCF $(12,8)$ calculations, having 8 MOs in the active space. In the paper body only 4 orbitals, the main carriers of unpaired spins, were represented. Here we represent all the 8 canonical CASSCF orbitals, including those that are mainly playing the doubly occupied subspace, with role in the improved account of the correlation effects.
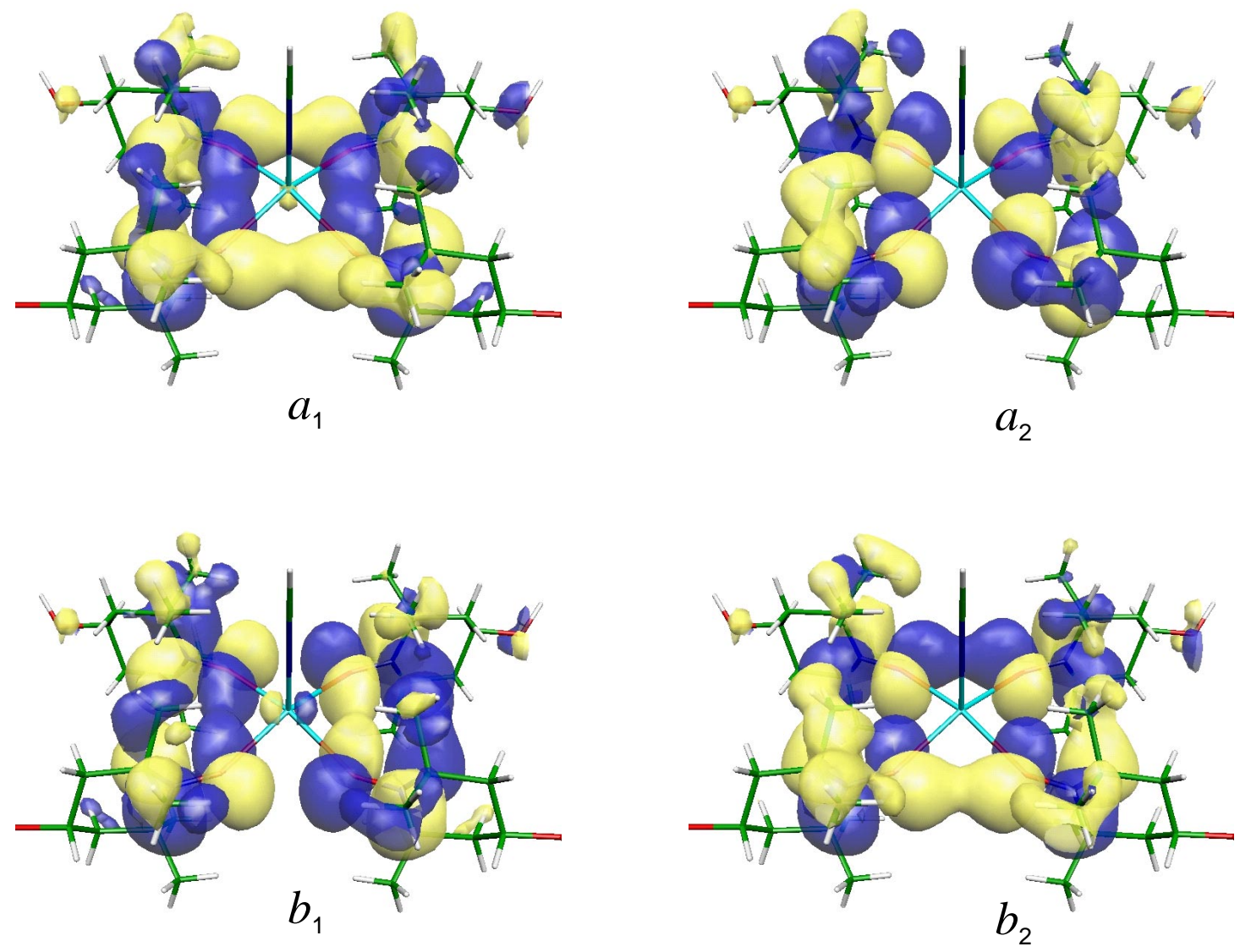

Figure II.1. The side view of the canonical CASSCF $(12,8)$ MOs related to the $\left|\pi_{\perp}^{*}\right\rangle$-type MOs of the TEMPOL units. The $\perp$ subscript refers to the perpendicular placement of the lobes with respect to the six member ring of each TEMPOL. With respect to the four radicals plane, these lobes are oriented parallel. 

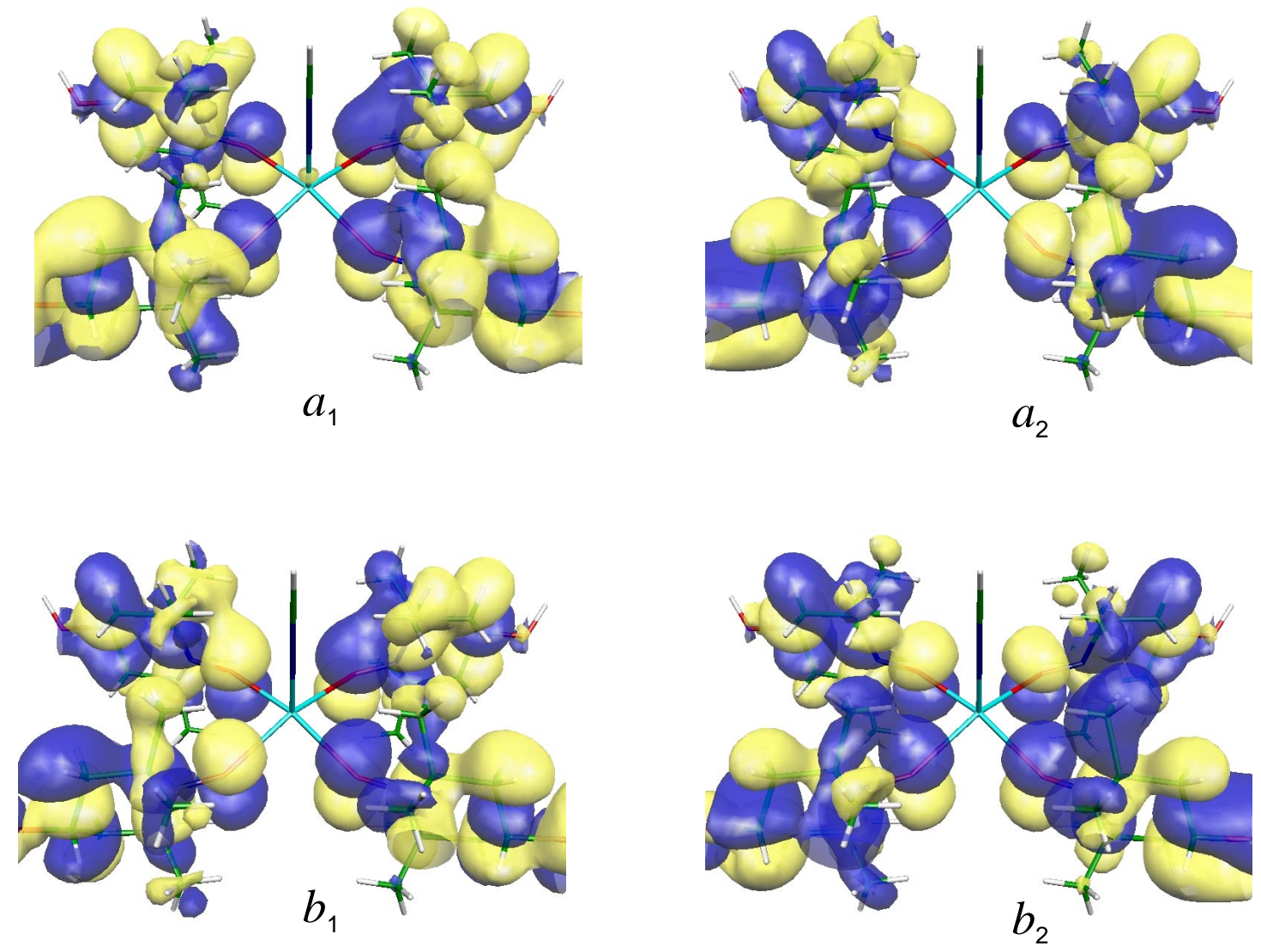

Figure II.2. The side view of the canonical CASSCF $(12,8)$ MOs related to the $\left|\pi_{\|}^{*-h y p e r}\right\rangle$-type MOs of the TEMPOL units. The $\|$ subscript refers to the parallel placement of the lobes with respect to the six member ring of each TEMPOL. The superscript hyper underlines their hyperconjugation character, consisting in $\pi^{*}$ antibonding like combination of $p$ AOs on the NO group, while the nitrogen components are in fact pertaining to a nonbonding symmetry combination of the $\sigma$ skeleton. The orbitals in this chart are mainly doubly occupied in all the configurations of the CASSCF results. 


\section{The Dipolar Spin Coupling Hamiltonian.}

In terms of polar coordinates the directory cosines are:

$$
\left\{\lambda_{A B}^{(x)}, \lambda_{A B}^{(y)}, \lambda_{A B}^{(z)}\right\}=\left\{\operatorname{Sin}\left(\theta_{A B}\right) \operatorname{Cos}\left(\varphi_{A B}\right), \operatorname{Sin}\left(\theta_{A B}\right) \operatorname{Sin}\left(\varphi_{A B}\right), \operatorname{Cos}\left(\theta_{A B}\right)\right\}
$$

The workable formula of dipolar interaction, in terms of spin ladder and projection operators, obtains as follows:

$$
\begin{aligned}
& \hat{H}_{d i p}^{A B}=D_{A B}\left[\left(\operatorname{Cos}\left(\theta_{A B}\right)^{2}-\frac{1}{3}\right)\left(\hat{S}_{A}^{(z)} \hat{S}_{B}^{(z)}-\frac{1}{4} \hat{S}_{A}^{(+)} \hat{S}_{B}^{(-)}-\frac{1}{4} \hat{S}_{A}^{(-)} \hat{S}_{B}^{(+)}\right)\right. \\
& +\frac{1}{4} \operatorname{Sin}\left(2 \theta_{A B}\right) \operatorname{Exp}\left(+\mathbf{i} \varphi_{A B}\right)\left(\hat{S}_{A}^{(z)} \hat{S}_{B}^{(-)}+\hat{S}_{A}^{(-)} \hat{S}_{B}^{(z)}\right)+\frac{1}{4} \operatorname{Sin}\left(2 \theta_{A B}\right) \operatorname{Exp}\left(-\mathbf{i} \varphi_{A B}\right)\left(\hat{S}_{A}^{(z)} \hat{S}_{B}^{(+)}+\hat{S}_{A}^{(+)} \hat{S}_{B}^{(z)}\right) \\
& \left.+\frac{1}{4} \operatorname{Sin}\left(\theta_{A B}\right)^{2} \operatorname{Exp}\left(+2 \mathbf{i} \varphi_{A B}\right)\left(\hat{S}_{A}^{(-)} \hat{S}_{B}^{(-)}+\hat{S}_{A}^{(-)} \hat{S}_{B}^{(-)}\right)+\frac{1}{4} \operatorname{Sin}\left(\theta_{A B}\right)^{2} \operatorname{Exp}\left(-2 \mathbf{i} \varphi_{A B}\right)\left(\hat{S}_{A}^{(+)} \hat{S}_{B}^{(+)}+\hat{S}_{A}^{(+)} \hat{S}_{B}^{(+)}\right)\right]
\end{aligned}
$$

The above formula is equivalent (except a factor of 3 , transferred here at the $D_{A B}$ parameter) to the 6.5 expansion in A. Bencini, D. Gatteschi, EPR of Exchange Coupled Sytems, Springer-Verlag, Berlin 1990.

The quantitative estimation of dipolar magnitude, shortcut formulas are:

$D_{A B}=38940.41 g^{2} /\left(r_{A B}\right)^{3}$ for $D_{A B}=$ in $\mathrm{MHz}$ and $r_{A B}$ in $\AA$.

$D_{A B}=27822.05 g /\left(r_{A B}\right)^{3}$ for $D_{A B}=$ in Gauss and $r_{A B}$ in $\AA$.

$D_{A B}=1.299 g^{2} /\left(r_{A B}\right)^{3}$ for $D_{A B}=$ in $\mathrm{cm}^{-1}$ and $r_{A B}$ in $\AA$.

Note that some other literature conventions may assign expressions affected by certain different factors. E.g. in Bencini \& Gatteschi monograph, the factor for conversion to $\mathrm{cm}^{-1}$ is three times smaller than in (A.III.3c), namely 0.433 (formula 3.73 page 76 ). The situation is due to the above noted transfer of the factor 3 from Hamiltonian expansion to be included inside the parameter itself. The reason of the formal definitions adopted here is to work with master formulas that yield at end the ZFS in the customary form $D\left(S_{z}^{2}-S(S+1) / 3\right)$. When dealing with dimers only, another used convention incorporates a $1 / 2$ factor. This is due to the fact that, working with the Hamiltonian III.1 for a dimer made of $1 / 2$ spins, the nominal triplet ZFS parameter 
is $D_{T}=D_{A B} / 2$. Of course, if properly marked and consistently used the adopted convention does not affect the final results.

The basis of spin projections and the corresponding labeling of the configurations is given in the next table.

Table III.1.

\begin{tabular}{|c|c|c|c|c|}
\hline$M_{S}=-2$ & $M_{S}=-1$ & $M_{S}=0$ & $M_{S}=+1$ & $M_{S}=+2$ \\
\hline \multirow[t]{6}{*}{ 1) $|\beta \beta \beta \beta|$} & 2) $|\alpha \beta \beta \beta|$ & 6) $|\alpha \beta \alpha \beta|$ & 12) $|\alpha \beta \alpha \alpha|$ & \multirow[t]{6}{*}{ 16) $|\alpha \alpha \alpha \alpha|$} \\
\hline & 3) $|\beta \beta \alpha \beta|$ & 7) $|\alpha \beta \beta \alpha|$ & 13) $|\alpha \alpha \alpha \beta|$ & \\
\hline & 4) $|\beta \beta \beta \alpha|$ & 8) $|\beta \beta \alpha \alpha|$ & 14) $|\alpha \alpha \beta \alpha|$ & \\
\hline & 5) $|\beta \alpha \beta \beta|$ & 9) $|\alpha \alpha \beta \beta|$ & 15) $|\beta \alpha \alpha \alpha|$ & \\
\hline & & 10) $|\beta \alpha \alpha \beta|$ & & \\
\hline & & 11) $|\beta \alpha \beta \alpha|$ & & \\
\hline
\end{tabular}

The transformation of the above basis set to the spin blocks ( 2 singlets, 3 triplets and 1 quintet) is performed with the following matrix:

$\boldsymbol{t}=\left(\begin{array}{cccccccccccccccc}\mathbf{0} & \mathbf{0} & \mathbf{0} & \mathbf{0} & \mathbf{0} & \mathbf{0} & \frac{1}{2} & -\frac{1}{2} & -\frac{1}{2} & \frac{1}{2} & \mathbf{0} & \mathbf{0} & \mathbf{0} & \mathbf{0} & \mathbf{0} & \mathbf{0} \\ \mathbf{0} & \mathbf{0} & \mathbf{0} & \mathbf{0} & \mathbf{0} & \frac{1}{\sqrt{3}} & -\frac{1}{2 \sqrt{3}} & -\frac{1}{2 \sqrt{3}} & -\frac{1}{2 \sqrt{3}} & -\frac{1}{2 \sqrt{3}} & \frac{1}{\sqrt{3}} & \mathbf{0} & \mathbf{0} & \mathbf{0} & \mathbf{0} & \mathbf{0} \\ \mathbf{0} & \frac{1}{2} & -\frac{1}{2} & \frac{1}{2} & -\frac{1}{2} & \mathbf{0} & \mathbf{0} & \mathbf{0} & \mathbf{0} & \mathbf{0} & \mathbf{0} & \mathbf{0} & \mathbf{0} & \mathbf{0} & \mathbf{0} & \mathbf{0} \\ \mathbf{0} & \mathbf{0} & \mathbf{0} & \mathbf{0} & \mathbf{0} & \mathbf{0} & \frac{1}{\sqrt{2}} & \mathbf{0} & \mathbf{0} & -\frac{1}{\sqrt{2}} & \mathbf{0} & \mathbf{0} & \mathbf{0} & \mathbf{0} & \mathbf{0} & \mathbf{0} \\ \mathbf{0} & \mathbf{0} & \mathbf{0} & \mathbf{0} & \mathbf{0} & \mathbf{0} & \mathbf{0} & \mathbf{0} & \mathbf{0} & \mathbf{0} & \mathbf{0} & \frac{1}{2} & -\frac{1}{2} & \frac{1}{2} & -\frac{1}{2} & \mathbf{0} \\ \mathbf{0} & -\frac{1}{2} & \frac{1}{2} & \frac{1}{2} & -\frac{1}{2} & \mathbf{0} & \mathbf{0} & \mathbf{0} & \mathbf{0} & \mathbf{0} & \mathbf{0} & \mathbf{0} & \mathbf{0} & \mathbf{0} & \mathbf{0} & \mathbf{0} \\ \mathbf{0} & \mathbf{0} & \mathbf{0} & \mathbf{0} & \mathbf{0} & \mathbf{0} & \mathbf{0} & \frac{1}{\sqrt{2}} & -\frac{1}{\sqrt{2}} & \mathbf{0} & \mathbf{0} & \mathbf{0} & \mathbf{0} & \mathbf{0} & \mathbf{0} & \mathbf{0} \\ \mathbf{0} & \mathbf{0} & \mathbf{0} & \mathbf{0} & \mathbf{0} & \mathbf{0} & \mathbf{0} & \mathbf{0} & \mathbf{0} & \mathbf{0} & \mathbf{0} & \frac{1}{2} & -\frac{1}{2} & -\frac{1}{2} & \frac{1}{2} & \mathbf{0} \\ \mathbf{0} & \frac{1}{2} & \frac{1}{2} & -\frac{1}{2} & -\frac{1}{2} & \mathbf{0} & \mathbf{0} & \mathbf{0} & \mathbf{0} & \mathbf{0} & \mathbf{0} & \mathbf{0} & \mathbf{0} & \mathbf{0} & \mathbf{0} & \mathbf{0} \\ \mathbf{0} & \mathbf{0} & \mathbf{0} & \mathbf{0} & \mathbf{0} & \frac{1}{\sqrt{2}} & \mathbf{0} & \mathbf{0} & \mathbf{0} & \mathbf{0} & -\frac{1}{\sqrt{2}} & \mathbf{0} & \mathbf{0} & \mathbf{0} & \mathbf{0} & \mathbf{0} \\ \mathbf{0} & \mathbf{0} & \mathbf{0} & \mathbf{0} & \mathbf{0} & \mathbf{0} & \mathbf{0} & \mathbf{0} & \mathbf{0} & \mathbf{0} & \mathbf{0} & \frac{1}{2} & \frac{1}{2} & -\frac{1}{2} & -\frac{1}{2} & \mathbf{0} \\ \mathbf{1} & \mathbf{0} & \mathbf{0} & \mathbf{0} & \mathbf{0} & \mathbf{0} & \mathbf{0} & \mathbf{0} & \mathbf{0} & \mathbf{0} & \mathbf{0} & \mathbf{0} & \mathbf{0} & \mathbf{0} & \mathbf{0} & \mathbf{0} \\ \mathbf{0} & \frac{1}{2} & \frac{1}{2} & \frac{1}{2} & \frac{1}{2} & \mathbf{0} & \mathbf{0} & \mathbf{0} & \mathbf{0} & \mathbf{0} & \mathbf{0} & \mathbf{0} & \mathbf{0} & \mathbf{0} & \mathbf{0} & \mathbf{0} \\ \mathbf{0} & \mathbf{0} & \mathbf{0} & \mathbf{0} & \mathbf{0} & \frac{1}{\sqrt{5}} & \frac{1}{\sqrt{5}} & \frac{1}{\sqrt{6}} & \frac{1}{\sqrt{5}} & \frac{1}{\sqrt{6}} & \frac{1}{\sqrt{6}} & \mathbf{0} & \mathbf{0} & \mathbf{0} & \mathbf{0} & \mathbf{0} \\ \mathbf{0} & \mathbf{0} & \mathbf{0} & \mathbf{0} & \mathbf{0} & \mathbf{0} & \mathbf{0} & \mathbf{0} & \mathbf{0} & \mathbf{0} & \mathbf{0} & \frac{1}{2} & \frac{1}{2} & \frac{1}{2} & \frac{1}{2} & \mathbf{0} \\ \mathbf{0} & \mathbf{0} & \mathbf{0} & \mathbf{0} & \mathbf{0} & \mathbf{0} & \mathbf{0} & \mathbf{0} & \mathbf{0} & \mathbf{0} & \mathbf{0} & \mathbf{0} & \mathbf{0} & \mathbf{0} & \mathbf{0} & \mathbf{1}\end{array}\right)$

(III.4)

In the above matrix each row corresponds to a spin state component. E.g. the first row defines the first singlet state. Taking the definition of the configurations from the 
above table (the configurations of non-zero coefficients no.7-10) the first singlet is exemplified as follows: $\mid S_{1}>=1 / 2(|\alpha \beta \beta \alpha|-|\beta \beta \alpha \alpha|-|\alpha \alpha \beta \beta|+|\beta \alpha \alpha \beta|)$

A convenient choice of mutual angular parameters, used when the dipolar Hamiltonian III. 2 is applied, is given in the following table. The $z$ axis was chosen perpendicular to the plane of the spin rectangle.

Table III.2. Angular parameters of mutual positions of the spin dipoles.

\begin{tabular}{l|l|l|l|l|l|l}
$\mathrm{A}-\mathrm{B}$ & $\mathbf{1 - 3}$ & $\mathbf{2 - 4}$ & $\mathbf{1 - 4}$ & $\mathbf{2 - 3}$ & $\mathbf{1 - 2}$ & $\mathbf{3 - 4}$ \\
\hline$\theta_{\mathrm{AB}}$ & $90^{\circ}$ & $90^{\circ}$ & $90^{\circ}$ & $90^{\circ}$ & $90^{\circ}$ & $90^{\circ}$ \\
\hline$\varphi_{\mathrm{AB}}$ & $-u$ & $u$ & 0 & 0 & 0 & 0
\end{tabular}

Denoting by $R_{a}$ and $R_{b}$ the edges of the rectangle, the angle denoted $u$ corresponds to $u=\operatorname{ArcTan}\left(R_{a} / R_{b}\right)$. In the discussed system $u \sim 42.5^{\circ}$. For the sake of topological simplification under the semiquantitative circumstances, at the end of the handling the resulted formulas were considered for the $u \sim 45^{\circ}$ value.

Within the above coordinate choice, the three triplets show the following block matrices:

$$
\begin{aligned}
& \mathbf{H}_{T_{1}}=\left(\begin{array}{ccc}
-\frac{D_{a}}{12}-\frac{D_{b}}{12}+\frac{D_{d}}{12} & 0 & \frac{D_{a}}{4}-\frac{D_{b}}{4}-\frac{D_{d}}{4} \operatorname{Cos}(2 u) \\
0 & \frac{D_{a}}{6}+\frac{D_{b}}{6}-\frac{D_{d}}{6} & 0 \\
\frac{D_{a}}{4}-\frac{D_{b}}{4}-\frac{D_{d}}{4} \operatorname{Cos}(2 u) & 0 & -\frac{D_{a}}{12}-\frac{D_{b}}{12}+\frac{D_{d}}{12}
\end{array}\right) \\
& \mathbf{H}_{T_{2}}=\left(\begin{array}{ccc}
-\frac{D_{a}}{12}+\frac{D_{b}}{12}-\frac{D_{d}}{12} & 0 & \frac{D_{a}}{4}+\frac{D_{b}}{4}+\frac{D_{d}}{4} \operatorname{Cos}(2 u) \\
0 & \frac{D_{a}}{6}-\frac{D_{b}}{6}+\frac{D_{d}}{6} & 0 \\
\frac{D_{a}}{4}+\frac{D_{b}}{4}+\frac{D_{d}}{4} \operatorname{Cos}(2 u) & 0 & -\frac{D_{a}}{12}+\frac{D_{b}}{12}-\frac{D_{d}}{12}
\end{array}\right) \\
& \mathbf{H}_{T_{3}}=\left(\begin{array}{ccc}
\frac{D_{a}}{12}-\frac{D_{b}}{12}-\frac{D_{d}}{12} & -\frac{D_{a}}{6}+\frac{D_{b}}{6}+\frac{D_{d}}{6} & -\frac{D_{a}}{4}-\frac{D_{b}}{4}+\frac{D_{d}}{4} \operatorname{Cos}(2 u) \\
0 & 0 & 0 \\
-\frac{D_{a}}{4}-\frac{D_{b}}{4}+\frac{D_{d}}{4} \operatorname{Cos}(2 u) & 0 & \frac{D_{b}}{12}-\frac{D_{d}}{12}
\end{array}\right)
\end{aligned}
$$


Between the second and third triplet there is a non-diagonal block:

$$
\mathbf{h}_{T_{2} T_{3}}=\left(\begin{array}{ccc}
0 & 0 & -\mathbf{i} \frac{D_{d}}{4} \operatorname{Sin}(2 u) \\
0 & 0 & 0 \\
\mathbf{i} \frac{D_{d}}{4} \operatorname{Sin}(2 u) & 0 & 0
\end{array}\right)
$$

The quintet is:

$$
\mathbf{H}_{Q}=\left(\begin{array}{ccccc}
-\frac{1}{6}\left(D_{a}+D_{b}+D_{d}\right) & 0 & \frac{1}{2 \sqrt{6}}\left(D_{a}-D_{b}+\operatorname{Cos}(2 u) D_{d}\right) & 0 & 0 \\
0 & \frac{1}{12}\left(D_{a}+D_{b}+D_{d}\right) & 0 & \frac{1}{4}\left(D_{a}-D_{b}+\operatorname{Cos}(2 u) D_{d}\right) & 0 \\
\frac{1}{2 \sqrt{6}}\left(D_{a}-D_{b}+\operatorname{Cos}(2 u) D_{d}\right) & 0 & \frac{1}{6}\left(D_{a}+D_{b}+D_{d}\right) & 0 & \frac{1}{2 \sqrt{6}}\left(D_{a}-D_{b}+\operatorname{Cos}(2 u) D_{d}\right) \\
0 & \frac{1}{4}\left(D_{a}-D_{b}+\operatorname{Cos}(2 u) D_{d}\right) & 0 & \frac{1}{12}\left(D_{a}+D_{b}+D_{d}\right) & 0 \\
0 & 0 & \frac{1}{2 \sqrt{6}}\left(D_{a}-D_{b}+\operatorname{Cos}(2 u) D_{d}\right) & 0 & -\frac{1}{6}\left(D_{a}+D_{b}+D_{d}\right)
\end{array}\right)
$$

(III.9)

For the sake of completeness we present also the block of singlet exchange energy

$$
\mathbf{H}_{S}=\left(\begin{array}{cc}
3 J_{d} & \sqrt{3} J_{a}-\sqrt{3} J_{b} \\
\sqrt{3} J_{a}-\sqrt{3} J_{b} & 2 J_{a}+2 J_{b}-J_{d}
\end{array}\right)
$$

and the singlet-quintet and triplet-quintet ZFS nondiagonal blocks.

$$
\begin{aligned}
\mathbf{h}_{S Q} & =\left(\begin{array}{ccc}
\frac{1}{4}\left(D_{a}+D_{b}\right) & \frac{1}{4 \sqrt{3}}\left(-D_{a}+D_{b}+2 \operatorname{Cos}(2 u) D_{d}\right) \\
0 & 0 \\
\frac{1}{2 \sqrt{6}}\left(-D_{a}+D_{b}\right) & \frac{1}{6 \sqrt{2}}\left(D_{a}+D_{b}-2 D_{d}\right) \\
\frac{1}{4}\left(D_{a}+D_{b}\right) & \frac{1}{4 \sqrt{3}}\left(-D_{a}+D_{b}+2 \operatorname{Cos}(2 u) D_{d}\right)
\end{array}\right) \\
\mathbf{h}_{T_{i} Q} & =\left(\begin{array}{ccc}
0 & -\mathbf{i} \frac{D_{d}}{2 \sqrt{2}} \operatorname{Sin}(2 u) & 0 \\
0 & 0 & -\mathbf{i} \frac{D_{d}}{4} \operatorname{Sin}(2 u) \\
0 & 0 & 0 \\
-\mathbf{i} \frac{D_{d}}{4} \operatorname{Sin}(2 u) & 0 & 0 \\
0 & -\mathbf{i} \frac{D_{d}}{2 \sqrt{2}} \operatorname{Sin}(2 u) & 0
\end{array}\right)
\end{aligned}
$$


Given the large separation of spin states, due to the large $J$ absolute values as compared to the $D$ ones (the $D$ are in the range of $10^{-2} \mathrm{~cm}^{-1}$ ), the nondiagonal blocks (outlined here only for the sake of the completeness) are not effectively important.

Taken at $u=45^{\circ}$ the triplet matrices can be diagonalized to:

T1:

$\left(\begin{array}{ccc}\frac{D_{a}}{6}-\frac{D_{b}}{3}+\frac{D_{d}}{12} & 0 & 0 \\ 0 & \frac{D_{a}}{6}+\frac{D_{b}}{6}-\frac{D_{d}}{6} & 0 \\ 0 & 0 & -\frac{D_{a}}{3}+\frac{D_{b}}{6}+\frac{D_{d}}{12}\end{array}\right)$

T2:

$\left(\begin{array}{ccc}\frac{D_{a}}{6}+\frac{D_{b}}{3}-\frac{D_{d}}{12} & 0 & 0 \\ 0 & \frac{D_{a}}{6}-\frac{D_{b}}{6}+\frac{D_{d}}{6} & 0 \\ 0 & 0 & -\frac{D_{a}}{3}-\frac{D_{b}}{6}-\frac{D_{d}}{12}\end{array}\right)$

T3:

$\left(\begin{array}{ccc}-\frac{D_{a}}{6}-\frac{D_{b}}{3}-\frac{D_{d}}{12} & 0 & 0 \\ 0 & -\frac{D_{a}}{6}+\frac{D_{b}}{6}+\frac{D_{d}}{6} & 0 \\ 0 & 0 & \frac{D_{a}}{3}+\frac{D_{b}}{6}-\frac{D_{d}}{12}\end{array}\right)$

These eigenvalues yield the ZFS expressions used in the paper.

The analytical handling of the Hamiltonian operator and matrices was made with programs written in the frame of computer package Mathematica. (Stephen Wolfram, Mathematica; Cambridge University, Press: Cambridge, 1999). 


\section{Crystallographic Data.}

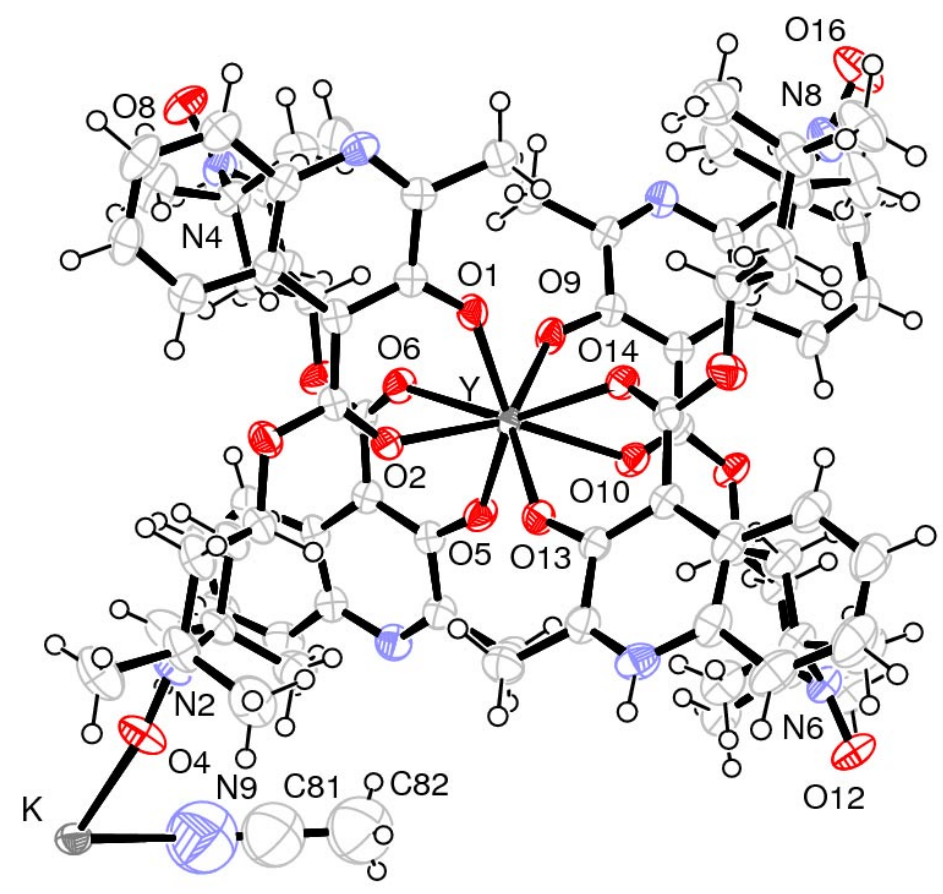

Figure IV. 1. Ortep diagram of $1 \cdot 2 \mathrm{CH}_{3} \mathrm{CN} \cdot 1 / 4 \mathrm{H}_{2} \mathrm{O}$ with thermal ellipsoids at the $50 \%$ probability level. The solvent molecules are omitted for clarity. 


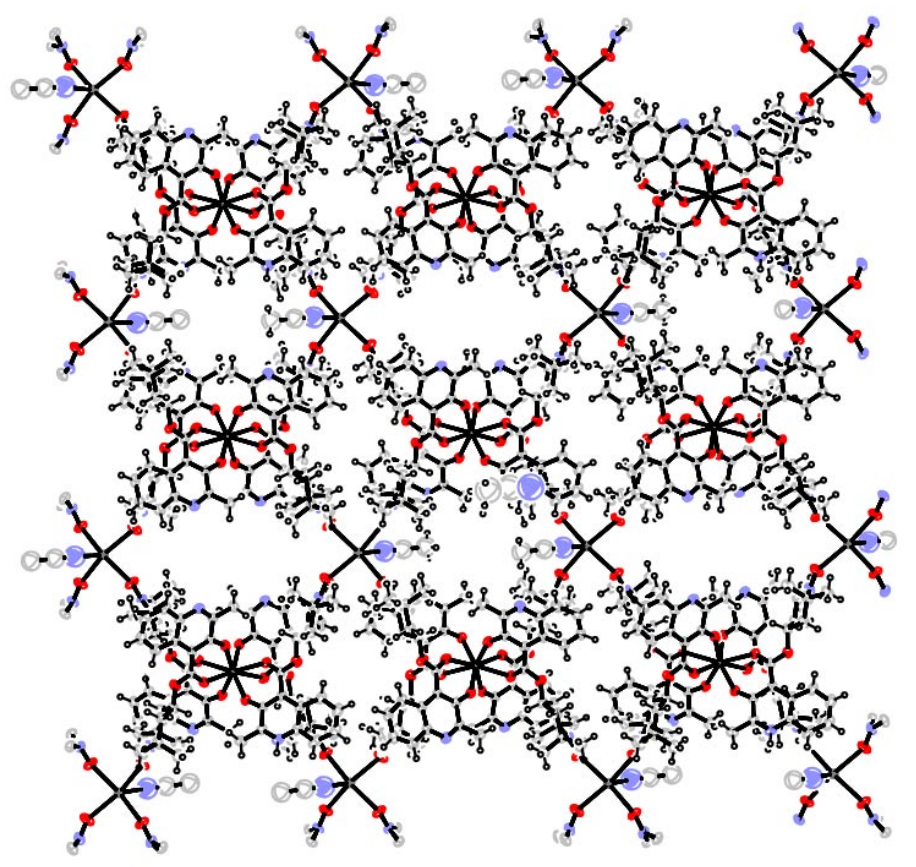

Figure IV.2. Packing diagram for the complex $1 \cdot 2 \mathrm{CH}_{3} \mathrm{CN} \cdot 1 / 4 \mathrm{H}_{2} \mathrm{O}$. 
Table IV.1. Atomic coordinates ( $\times 10^{4}$ ) and equivalent isotropic displacement parameters $\left(\AA^{2} \times 10^{3}\right)$ for $\mathrm{K}\left[\mathrm{Y}(\mathrm{QT})_{4}\left(\mathrm{CH}_{3} \mathrm{CN}\right)\right] \cdot 2 \mathrm{CH}_{3} \mathrm{CN} \cdot 1 / 4 \mathrm{H}_{2} \mathrm{O}$. U(eq) is defined as one third of the trace of the orthogonalized $\mathrm{U}_{\mathrm{ij}}$ tensor.

\begin{tabular}{|c|c|c|c|c|}
\hline & $\mathrm{x}$ & $\mathrm{y}$ & $\mathrm{z}$ & $\mathrm{U}(\mathrm{eq})$ \\
\hline $\mathrm{Y}(1)$ & $7435(1)$ & $45(1)$ & $2492(1)$ & $26(1)$ \\
\hline $\mathrm{K}(1)$ & 1959(1) & $-4304(1)$ & $2337(1)$ & $40(1)$ \\
\hline $\mathrm{O}(1)$ & $7211(2)$ & 186(2) & $1458(1)$ & $34(1)$ \\
\hline $\mathrm{O}(2)$ & $5906(2)$ & $-655(2)$ & $1724(1)$ & $30(1)$ \\
\hline $\mathrm{O}(3)$ & $4630(2)$ & $-1386(2)$ & $842(1)$ & $38(1)$ \\
\hline $\mathrm{O}(4)$ & $2539(2)$ & $-2981(2)$ & $1954(2)$ & $50(1)$ \\
\hline $\mathrm{N}(1)$ & $6537(2)$ & $-205(2)$ & $-364(2)$ & $33(1)$ \\
\hline $\mathrm{N}(2)$ & $3042(2)$ & $-2602(2)$ & $1702(2)$ & $36(1)$ \\
\hline$C(1)$ & 7833(3) & $826(3)$ & $604(2)$ & $42(1)$ \\
\hline$C(2)$ & $6977(3)$ & 116(3) & $316(2)$ & $30(1)$ \\
\hline$C(3)$ & $5733(3)$ & $-843(3)$ & $-629(2)$ & $32(1)$ \\
\hline $\mathrm{C}(4)$ & $5316(3)$ & $-1182(3)$ & $-1363(2)$ & $42(1)$ \\
\hline$C(5)$ & $4510(3)$ & $-1816(3)$ & $-1687(2)$ & $47(1)$ \\
\hline$C(6)$ & $4114(3)$ & $-2105(3)$ & $-1281(2)$ & $44(1)$ \\
\hline$C(7)$ & 4501(3) & $-1782(3)$ & $-555(2)$ & $39(1)$ \\
\hline $\mathrm{C}(8)$ & $5339(3)$ & $-1132(3)$ & $-196(2)$ & $27(1)$ \\
\hline $\mathrm{C}(9)$ & $5823(3)$ & $-761(2)$ & $562(2)$ & $26(1)$ \\
\hline$C(10)$ & $6677(3)$ & $-157(3)$ & $818(2)$ & $28(1)$ \\
\hline $\mathrm{C}(11)$ & $5484(3)$ & $-917(3)$ & $1090(2)$ & $26(1)$ \\
\hline$C(12)$ & 1661(3) & $-2202(3)$ & $1037(3)$ & $60(2)$ \\
\hline $\mathrm{C}(13)$ & $2749(3)$ & $-967(3)$ & $2139(3)$ & $62(2)$ \\
\hline$C(14)$ & 2674(3) & $-1841(3)$ & $1479(2)$ & $41(1)$ \\
\hline$C(15)$ & $3210(3)$ & $-1640(3)$ & $1026(2)$ & $41(1)$ \\
\hline$C(16)$ & $4233(3)$ & $-1541(3)$ & $1341(2)$ & $34(1)$ \\
\hline $\mathrm{C}(17)$ & $4475(3)$ & $-2444(3)$ & $1422(2)$ & $38(1)$ \\
\hline $\mathrm{C}(18)$ & $4040(3)$ & $-2703(3)$ & 1901(2) & $35(1)$ \\
\hline $\mathrm{C}(19)$ & $4503(3)$ & $-2091(3)$ & $2680(2)$ & $54(1)$ \\
\hline$C(20)$ & $4088(3)$ & $-3738(3)$ & 1781(3) & $58(1)$ \\
\hline $\mathrm{O}(5)$ & $7336(2)$ & $-858(2)$ & 3094(1) & $37(1)$ \\
\hline $\mathrm{O}(6)$ & $7376(2)$ & $-1633(2)$ & $1766(1)$ & $34(1)$ \\
\hline
\end{tabular}




\begin{tabular}{|c|c|c|c|c|}
\hline $\mathrm{O}(7)$ & $7464(2)$ & $-3160(2)$ & 1382(1) & 41(1) \\
\hline $\mathrm{O}(8)$ & $7717(2)$ & $-4316(2)$ & $-1287(2)$ & $51(1)$ \\
\hline $\mathrm{N}(3)$ & $6820(2)$ & $-2670(2)$ & $3649(2)$ & $42(1)$ \\
\hline $\mathrm{N}(4)$ & $7670(2)$ & $-4003(2)$ & $-654(2)$ & $34(1)$ \\
\hline $\mathrm{C}(21)$ & 7193(3) & $-987(3)$ & $4303(2)$ & $53(1)$ \\
\hline $\mathrm{C}(22)$ & $7038(3)$ & $-1848(3)$ & $3642(2)$ & $36(1)$ \\
\hline $\mathrm{C}(23)$ & $6667(3)$ & $-3463(3)$ & $3035(2)$ & $36(1)$ \\
\hline$C(24)$ & $6388(3)$ & $-4325(3)$ & $3065(3)$ & $48(1)$ \\
\hline $\mathrm{C}(25)$ & $6164(3)$ & $-5144(3)$ & $2484(3)$ & $51(1)$ \\
\hline$C(26)$ & $6232(3)$ & $-5129(3)$ & $1856(3)$ & $44(1)$ \\
\hline $\mathrm{C}(27)$ & $6523(3)$ & $-4298(3)$ & $1814(2)$ & $36(1)$ \\
\hline $\mathrm{C}(28)$ & $6762(3)$ & $-3431(3)$ & $2409(2)$ & $31(1)$ \\
\hline $\mathrm{C}(29)$ & $7047(3)$ & $-2523(3)$ & $2413(2)$ & $29(1)$ \\
\hline $\mathrm{C}(30)$ & $7141(3)$ & $-1710(3)$ & $3024(2)$ & $29(1)$ \\
\hline $\mathrm{C}(31)$ & $7283(3)$ & $-2378(3)$ & $1841(2)$ & $29(1)$ \\
\hline $\mathrm{C}(32)$ & $8679(3)$ & $-5091(3)$ & $-391(2)$ & $53(1)$ \\
\hline$C(33)$ & $9312(3)$ & $-3377(3)$ & $49(3)$ & $61(2)$ \\
\hline $\mathrm{C}(34)$ & $8470(3)$ & $-4083(3)$ & $-102(2)$ & $36(1)$ \\
\hline$C(35)$ & $8194(3)$ & $-3940(3)$ & $560(2)$ & $40(1)$ \\
\hline$C(36)$ & $7720(3)$ & $-3098(3)$ & $794(2)$ & $35(1)$ \\
\hline $\mathrm{C}(37)$ & $6863(3)$ & $-3200(3)$ & $187(2)$ & $36(1)$ \\
\hline $\mathrm{C}(38)$ & $7055(3)$ & $-3271(3)$ & $-486(2)$ & $36(1)$ \\
\hline C(39) & $7532(3)$ & $-2332(3)$ & $-429(2)$ & $49(1)$ \\
\hline $\mathrm{C}(40)$ & $6161(3)$ & $-3608(3)$ & $-1106(2)$ & $52(1)$ \\
\hline $\mathrm{O}(9)$ & $8839(2)$ & $-134(2)$ & $2426(1)$ & $34(1)$ \\
\hline $\mathrm{O}(10)$ & $8641(2)$ & $827(2)$ & $3669(1)$ & $35(1)$ \\
\hline $\mathrm{O}(11)$ & $9946(2)$ & $1319(2)$ & $4577(1)$ & $37(1)$ \\
\hline $\mathrm{O}(12)$ & $8827(2)$ & $2960(2)$ & $6897(1)$ & $45(1)$ \\
\hline$N(5)$ & $11105(2)$ & $450(2)$ & $2465(2)$ & $36(1)$ \\
\hline$N(6)$ & $9081(2)$ & $2578(2)$ & $6348(2)$ & $33(1)$ \\
\hline $\mathrm{C}(41)$ & $9816(3)$ & $-625(3)$ & $1512(2)$ & $48(1)$ \\
\hline$C(42)$ & $10250(3)$ & $76(3)$ & $2258(2)$ & $30(1)$ \\
\hline$C(43)$ & $11503(3)$ & $1086(3)$ & $3159(2)$ & $32(1)$ \\
\hline$C(44)$ & $12432(3)$ & 1491(3) & $3352(2)$ & $41(1)$ \\
\hline$C(45)$ & $12898(3)$ & $2116(3)$ & $4013(2)$ & $43(1)$ \\
\hline$C(46)$ & $12435(3)$ & $2359(3)$ & $4522(2)$ & $41(1)$ \\
\hline
\end{tabular}




\begin{tabular}{|c|c|c|c|c|}
\hline$C(47)$ & $11529(3)$ & $1985(3)$ & $4351(2)$ & $37(1)$ \\
\hline $\mathrm{C}(48)$ & 11023(3) & $1334(3)$ & $3663(2)$ & $29(1)$ \\
\hline$C(49)$ & $10065(3)$ & $908(3)$ & $3422(2)$ & $28(1)$ \\
\hline$C(50)$ & $9665(3)$ & $289(3)$ & $2711(2)$ & $29(1)$ \\
\hline $\mathrm{C}(51)$ & $9485(3)$ & $1016(3)$ & $3876(2)$ & $30(1)$ \\
\hline$C(52)$ & $10285(3)$ & 1982(3) & $7039(2)$ & $46(1)$ \\
\hline $\mathrm{C}(53)$ & $8833(3)$ & $917(3)$ & $6186(2)$ & $48(1)$ \\
\hline$C(54)$ & $9550(3)$ & 1731(3) & $6325(2)$ & $37(1)$ \\
\hline$C(55)$ & $10018(3)$ & 1519(3) & $5753(2)$ & $39(1)$ \\
\hline$C(56)$ & 9397(3) & $1507(3)$ & $5053(2)$ & $33(1)$ \\
\hline $\mathrm{C}(57)$ & $9107(3)$ & $2460(3)$ & $5172(2)$ & $38(1)$ \\
\hline $\mathrm{C}(58)$ & $8574(3)$ & $2735(3)$ & $5699(2)$ & $37(1)$ \\
\hline $\mathrm{C}(59)$ & $8528(3)$ & 3791(3) & $5936(2)$ & $53(1)$ \\
\hline$C(60)$ & $7599(3)$ & $2188(3)$ & $5392(2)$ & $52(1)$ \\
\hline $\mathrm{O}(13)$ & $6543(2)$ & $905(2)$ & $3058(1)$ & $33(1)$ \\
\hline $\mathrm{O}(14)$ & $7872(2)$ & 1711(2) & $2770(1)$ & $32(1)$ \\
\hline $\mathrm{O}(15)$ & $8018(2)$ & $3267(2)$ & $3112(2)$ & $42(1)$ \\
\hline $\mathrm{O}(16)$ & $10674(2)$ & $4404(2)$ & 2191(2) & $59(1)$ \\
\hline $\mathrm{N}(7)$ & $5612(2)$ & $2670(3)$ & $4153(2)$ & $41(1)$ \\
\hline $\mathrm{N}(8)$ & $10057(2)$ & $4108(2)$ & $2399(2)$ & $42(1)$ \\
\hline$C(61)$ & $5078(3)$ & 1001(3) & $3550(2)$ & $53(1)$ \\
\hline$C(62)$ & $5741(3)$ & $1865(3)$ & $3741(2)$ & $35(1)$ \\
\hline$C(63)$ & $6228(3)$ & $3464(3)$ & $4331(2)$ & $39(1)$ \\
\hline$C(64)$ & $6100(3)$ & 4301(3) & $4827(2)$ & $54(1)$ \\
\hline$C(65)$ & $6681(4)$ & $5135(3)$ & $5051(3)$ & $61(2)$ \\
\hline$C(66)$ & 7401(4) & $5137(3)$ & $4771(2)$ & $56(1)$ \\
\hline$C(67)$ & $7547(3)$ & $4329(3)$ & $4283(2)$ & $45(1)$ \\
\hline$C(68)$ & $6950(3)$ & $3460(3)$ & $4040(2)$ & $33(1)$ \\
\hline$C(69)$ & $7065(3)$ & $2567(3)$ & $3553(2)$ & $30(1)$ \\
\hline$C(70)$ & 6491(3) & $1747(3)$ & $3439(2)$ & $28(1)$ \\
\hline $\mathrm{C}(71)$ & $7683(3)$ & $2453(3)$ & $3123(2)$ & $29(1)$ \\
\hline$C(72)$ & $9178(3)$ & $5328(3)$ & 2201(3) & $56(1)$ \\
\hline$C(73)$ & $8697(3)$ & $3647(3)$ & $1319(2)$ & $64(2)$ \\
\hline $\mathrm{C}(74)$ & $9099(3)$ & $4293(3)$ & $2110(2)$ & $43(1)$ \\
\hline$C(75)$ & $8516(3)$ & $4135(3)$ & $2540(2)$ & $43(1)$ \\
\hline$C(76)$ & $8598(3)$ & $3236(3)$ & $2671(2)$ & $37(1)$ \\
\hline
\end{tabular}




\begin{tabular}{lrrrr}
$\mathrm{C}(77)$ & $9586(3)$ & $3250(3)$ & $3063(2)$ & $41(1)$ \\
$\mathrm{C}(78)$ & $10246(3)$ & $3337(3)$ & $2675(2)$ & $43(1)$ \\
$\mathrm{C}(79)$ & $10178(3)$ & $2413(3)$ & $2034(3)$ & $57(1)$ \\
$\mathrm{C}(80)$ & $11230(3)$ & $3605(3)$ & $3178(3)$ & $60(2)$ \\
$\mathrm{N}(9)$ & $3611(6)$ & $-3926(6)$ & $3404(4)$ & $170(3)$ \\
$\mathrm{C}(81)$ & $4207(6)$ & $-3253(7)$ & $3853(5)$ & $140(3)$ \\
$\mathrm{C}(82)$ & $5013(5)$ & $-2479(6)$ & $4434(4)$ & $148(3)$ \\
$\mathrm{N}(10)$ & $10133(4)$ & $-8718(4)$ & $281(3)$ & $126(2)$ \\
$\mathrm{C}(83)$ & $10746(5)$ & $-9034(5)$ & $581(4)$ & $104(2)$ \\
$\mathrm{C}(84)$ & $11512(4)$ & $-9294(4)$ & $1002(3)$ & $93(2)$ \\
$\mathrm{C}(85)$ & $3235(8)$ & $2406(8)$ & $2368(6)$ & $244(6)$ \\
$\mathrm{C}(86)$ & $3900(9)$ & $3189(11)$ & $2849(7)$ & $221(5)$ \\
$\mathrm{N}(11)$ & $4153(7)$ & $4098(8)$ & $3167(5)$ & $238(4)$ \\
$\mathrm{O}(17)$ & 5000 & 0 & 5000 & $153(6)$ \\
& & & & \\
\hline
\end{tabular}

